\title{
Time to rationalize the use of antibiotics
}

Aisha Sajid ${ }^{a}$

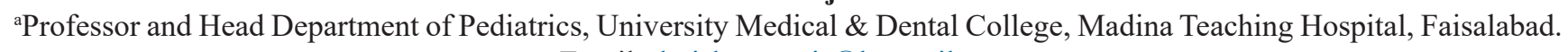
Email: draisha_tanvir@hotmail.com

Sajid A. Time to rationalize the use of antibiotics. Journal of University Medical \& Dental College. 2022;13(1):v-vi.

Antimicrobial drugs are considered as integral part of modern medicine and used to combat serious bacterial infections that may prove lethal otherwise. All life saving medical and surgical interventions, including surgery, chemotherapy, and organ transplant also rely upon the effective use of antibiotics. The darker side of the scenario is that the inappropriate use of antibiotics has become a serious issue all over the world, including inadequate dosage and duration, inappropriate use of broad spectrum antibiotics, and incomplete course of therapy. Inappropriate use of antibiotics across the globe has resulted in many serious consequences including not only the untoward side effects but also an alarming increase in antimicrobial resistance. Emergence of multidrug resistant microorganisms has worsened the situation. According to an international survey, Pakistan has been ranked third for the highest consumption of antibiotics among developing countries, and unfortunately, there has been $65 \%$ increase in the use of antibiotics from 200 to 2015 in Pakistan ${ }^{[1]}$.

Certain factors have been identified internationally as well as regionally that contribute towards the overuse and misuse of antibiotics. A review of literature reveals patient related factors like self medication, poor compliance towards prescription in terms of inadequate dosage, and inadequate duration of treatment. Many factors still are physicians dependent and if not followed, may result in suboptimal prescription like appropriate indication, choice of first line antibiotic, adequate dosage and duration of treatment ${ }^{[2]}$.

General public related factors that could have been identified in various regions of our country include poor knowledge, lack of education and awareness regarding antibiotics. Many patients consider antibiotics as miracle drug and prefer them to simple medication like analgesics, antipyretics and anti histamines for common colds and flu. Most of the drugs are easily available over the counter without prescription which is again contributing towards the magnitude of the problem. Certain patients leave the antibiotics on second or third day as their symptoms relive or due to unwanted side effects. Irrational prescription of antibiotics by quacks even for minor illnesses without indication is a common observation. Many patients force the physicians to take the prescription of their own choice either in the form of injectables or antibiotics ${ }^{[3,4,5]}$. Categorically, if we talk about pediatric age group, parents take the illness of their children more seriously and demand quick relief that may result in inappropriate drug of choice either by the treating physician or by the parents themselves. It is a common experience that as soon as the child gets febrile, parents start giving broad spectrum antibiotics by self-medication or even injectable antibiotics by quacks without adequate indication.

It has become a challenging task to overcome the factors inhibiting the Judicious and rational use of antimicrobials throughout the world. World Health Assembly (WHA) has endorsed The Global Action Plan to tackle the growing problem of antimicrobial resistance (AMR) in the 68th session held in Geneva in May 2015. It has defined the main five objectives ${ }^{[6]}$.

1. Improve awareness and understanding of antimicrobial resistance;

2. Strengthen knowledge through surveillance and research;

3. Reduce the incidence of infection;

4. Optimize the use of antimicrobial agents; and

5. Develop economic case for sustainable investment based on country needs and increase investment in new vaccines, diagnostics, and other interventions.

The government of Pakistan has launched the National Action Plan in accordance with the world health assembly to control the spread of antimicrobial resistance with defined objectives and strategies. One of the strategies is to update the regulations of human and veterinary use of antibiotic in all hospitals. We must understand our role as treating physicians and play our part in compliance with the national action plan. 


\section{Time to rationalize the use of antibiotics}

There is a dire need of public awareness campaigns for improving their understanding of this problem, to overcome the factors leading to misuse and overuse of antimicrobials, avoidance of self medication, quackery and increasing the compliance towards the doctor's prescription. Public awareness regarding prevention of infection by improving hygienic measures and promoting the use of vaccines is also important. Health personals must follow the clear boundaries for injectable antibiotics use based on appropriate indication. Hospitals may develop their own evidence based strategies of antibiotic choices according to the culture sensitivity results. Immediate policy is required at a national level for pharmaceuticals regarding the sale of antibiotics without prescription. The aim to rationalize the use of antibiotics can be achieved by active participation of all the sectors, including general public, policymakers, health care professionals, and institutions engaged in healthcare both in private and public sectors.

\section{REFERENCES:}

1. Klein EY, Van Boeckel TP, Martinez EM, Pant S, Gandra S, Levin SA, et al. Global increase and geographic convergence in antibiotic consumption between 2000 and 2015. Proceedings of the National Academy of Sciences. 2018;115(15):E3463-E3470.

2. Lushniak BD. Surgeon general's perspectives. Public Health Reports. 2014;129(4):314-316. Doi: 10.1177/003335491412900402

3. Gillani AH, Ji W, Hussain W, Imran A, Chang J, Yang C, et al. Antibiotic self-medication among non-medical university students in Punjab, Pakistan: a cross-sectional survey. International Journal of Environmental Research and Public Health. 2017;14(10):1152

4. Akhund R, Jamshed F, Jaffry HA, Hanif H, Fareed S. Knowledge and attitude of general Pakistani population towards antibiotic resistance. Cureus. 2019;11(3): e4266. Doi:10.7759/cureus.4266

5. Atif M, Asghar S, Mushtaq I, Malik I, Amin A, Babar ZU, et al. What drives inappropriate use of antibiotics? A mixed methods study from Bahawalpur, Pakistan. Infection and Drug Resistance. 2019;12:687-699. Doi: 10.2147/IDR. S189114

6. WHO. Antimicrobial resistance: A manual for developing national action plans; 2016 [cited 2020 July 15]. Available at http://apps.who.int/iris/bitstream/10665/204470/1/9789241549530 eng. 